\title{
Efeitos das Respostas de Observação Diferenciais sobre a Aprendizagem de Relações Condicionais com Estímulos Complexos
}

\author{
Jair Lopes Junior \\ Giovana Galvanin da Costa ${ }^{123}$ \\ Universidade Estadual Paulista/Bauru
}

\begin{abstract}
Resumo
Este estudo avaliou se relações entre os componentes do estímulo modelo complexo exerceriam controle condicional em tarefas de matching-to-sample simultâneo. $\mathrm{Na}$ Fase 1, 3 crianças com necessidades especiais de ensino foram expostas ao treino das relações A1B1 e A2B2 e ao teste das respectivas relações simétricas. Em seguida, as contingências de treino exigiram respostas de observação diferenciais que consistiram no estabelecimento de relações condicionais de identidade entre estímulos complexos (relações $\mathrm{AB}-\mathrm{AB}$ ) precedendo o acesso ao treino das relações condicionais ABX. Neste treino, diante de estímulos modelos complexos cujos componentes sustentavam condicionalidade treinada (A1B1 e A2B2), X1 foi o estímulo de escolha correto; X2 exerceu esta função quando os componentes do estímulo modelo não sustentavam tal relação (A1B2 e A2B1). Na Fase 2, ocorreria o treino PQ, testes QP e PQX que avaliariam a extensão do controle condicional definido pelas relações entre os estímulos $\mathrm{P}$ e Q. As três crianças registraram a aprendizagem das relações $\mathrm{AB}$, a emergência das relações simétricas e índices elevados de acerto nas respostas de observação diferenciais, ou seja, no estabelecimento das relações condicionais de identidade com estímulos complexos. Contudo, as três demonstraram relações de controle distintas das previstas no treino ABX, sendo, portanto, o experimento finalizado na Fase 1. Tais resultados sugerem uma independência funcional entre as habilidades discriminativas exigidas nas duas contingências de ensino de relações condicionais com estímulos modelo complexos.

Palavras-chave: Relações condicionais; estímulos complexos; respostas de observação diferenciais; crianças com necessidades educativas especiais.
\end{abstract}

Effects of Differential Observing Responses on the Learning of Conditional Relations with Complex Stimuli

\begin{abstract}
This study evaluated if relations between components of complex sample stimuli would have controlled conditional responding in identity and arbitrary simultaneous matching-to-sample tasks. In Phase 1, 3 children with educational special needs were trained to select stimulus $\mathrm{B} 1$ in the presence of stimulus A1, and select B2 in the presence of A2 (AB conditional relations); then symmetrical BA relations BA were tested. Afterwards they were exposed to differential observing response procedure that prompted children to make simultaneous identity matching responses with complex sample and comparison stimuli (AB-AB relations). In the sequence, the $\mathrm{ABX}$ conditional relations were trained. One stimulus in set $\mathrm{A}$ and another in set $\mathrm{B}$ appeared together as a sample, and two novel stimuli were the comparisons. Selection of X1 was reinforced if the two stimuli in the AB complex sample had been related in the previous training, and selection of X2 was reinforced if the components of AB sample had not been conditionally related. In Phase 2, PQ and QP conditional relations had been trained and tested, respectively. The aim of PQX tests was evaluated if selection of X1 and X2 would have been controlled by conditional or non-conditional relations between $\mathrm{P}$ and $\mathrm{Q}$ stimuli as complex sample. All children who learned $\mathrm{AB}$ conditional relations, showed $\mathrm{BA}$ symmetrical emergency, and obtained high accuracy level at differential observing response, namely they demonstrated $\mathrm{AB}-\mathrm{AB}$ identity matching-to-sample. Differently neither of them met learning criterion on ABX training. Then the experiment was stopped in Phase 1. In addition to literature data, these results demonstrated functional independency between discriminative skills required by the two teaching contingencies of conditional relations with complex stimuli.

Keywords: Conditional relations; complex stimuli; differential observing response; children with educational special needs.
\end{abstract}

A aprendizagem de relações arbitrárias entre estímulos (relational learning; cf. Green, Mackay, McIlvane, Saunders \& Soraci, 1990) constitui-se em repertório comportamental relevante para a interação com diversas contingências de

${ }^{1}$ Projeto parcialmente financiado pela Fundação de Amparo à Pesquisa do Estado de São Paulo (Proc. FAPESP 97/11462-0) e pelo PRONEX/FINEPCAPES.

${ }^{2}$ Bolsista IC/FAPESP.

${ }^{3}$ Endereço para correspondência: UNESP/FC-Departamento de Psicologia. Av. Eng. Luiz Edmundo C.Coube s/n., 17033 360, Bauru, SP. Fone: (14)2216087; Fax: (14)2216071. E-mail: jlopesjr@fc.unesp.br ensino. Pesquisas empíricas voltadas para a caracterização de processos comportamentais que definem a aprendizagem de relações entre estímulos, tanto quanto rotinas de avaliação clínica e educacional, fundamentam-se na utilização de diferentes procedimentos, dentre os quais o matching-to-sample (Dube, 1996; Dube \& Serna, 1998; Serna, Wilkinson \& McIlvane, 1998; Wilkinson, Dube \& McIlvane, 1998), em suas diferentes modalidades (simultâneo, com atraso, de identidade, arbitrário, com e sem correção; cf. Carter \& Werner, 1978; Cumming \& Berryman, 1965). Através do procedimento de matching-to-sample pode-se 
delinear, por exemplo, contingências de quatro termos (Lopes Jr. \& Matos, 1995; Sidman, 1986, 1994) nas quais as funções discriminativas $(\mathrm{S}+$ e $\mathrm{S}$ ) dos estímulos de escolha são definidas pelo estímulo modelo. Assim, diante dos estímulos de escolha B1 e B2, a seleção do estímulo B1 será correta com a apresentação (simultânea ou com atraso) do estímulo A1; por sua vez, diante destes mesmos estímulos de escolha, com a apresentação do estímulo A2 e não mais A1, a seleção do estímulo B2 passa a ser a correta ${ }^{4}$. Através do procedimento de matching-to-sample podem ser estabelecidas relações condicionais entre estímulos de uma mesma modalidade sensorial, tanto quanto de diferentes modalidades (Annet \& Leslie, 1995; Belanich \& Fields, 1999; DeGrandpre, Bickel \& Higgins, 1992; Dube, Green \& Serna, 1993; Green, 1990; L. Hayes, Tilley \& S. Hayes, 1988; O’Leary \& Bush, 1996; Tierney, DeLangy \& Braken, 1995).

A literatura registra investigações nas quais o estudo da aprendizagem relacional foi fundamentado na utilização do procedimento de matching-to-sample envolvendo estímulos (modelo e/ou escolha) complexos. Estímulos complexos são estímulos constituídos por mais de um elemento ( $R$. Stromer, McIlvane, Dube \& Mackay, 1993a; R. Stromer, McIlvane \& Serna, 1993b; Carpentier, Smeets \& BarnesHolmes, 2000).

Dois tipos de delineamentos experimentais são usualmente registrados nas pesquisas com estímulos complexos. Num primeiro tipo, cada elemento do estímulo modelo complexo pode exercer controle independente sobre a seleção de estímulos de escolha (Schenk, 1993; Smeets, Schenk \& Barnes, 1995; R. Stromer \& L. Stromer, 1990a, 1990b, 1992). De acordo com este primeiro tipo, teríamos o treino de relações condicionais nas quais a função de modelo seria exercida por estímulos complexos como A1B1 e A2B2. Seriam treinadas as seleções dos estímulos C1 e C2, diante dos estímulos A1B1 e A2B2, respectivamente (relações A1B1-C1 e A2B2-C2). Carpentier e colaboradores (2000) apontam que, considerados coletivamente, os principais resultados das pesquisas fundamentadas neste primeiro tipo de delineamento acusam que o treino das relações A1B1-C1 e A2B2-C2, na realidade, produz relações condicionais entre cada componente do estímulo modelo complexo e os respectivos estímulos de escolha definidos como corretos (A1-C1, B1-C1, A2-C2, B2-C2), bem como entre os componentes do estímulo modelo complexo (A1-B1 e A2-B2).

\footnotetext{
${ }^{4}$ Trata-se de uma caracterização do matching-to-sample condicional, modalidade utilizada no presente trabalho. A literatura registra trabalhos com análises comparativas entre essa modalidade e o matching-to-sample não-condicional (cf. Dube, McIlvane \& Green, 1992; Dube \& Serna, 1998).
}

De acordo com um segundo tipo de delineamento, os estímulos modelo complexos funcionam como compostos, a saber, não há um controle independente de cada componente (elemento) do estímulo modelo sobre a função discriminativa dos estímulos de escolha. Diferentemente, são as relações mantidas entre os componentes do estímulo modelo complexo que definem qual será o estímulo de escolha correto (Bush, Sidman \& de Rose, 1989; Gatch \& Osborne, 1989; Kennedy \& Laitinen, 1988; Lynch \& Green, 1991; Markham \& Dougher, 1993; Perez-Gonzalez, 1994) 5 .

Os experimentos de Perez-Gonzalez (1994) não apenas ilustram este segundo tipo de delineamento, como igualmente sustentam pertinência para o estudo descrito no presente artigo. Através da utilização do procedimento de matchingto-sample simultâneo com desenhos arbitrários, PerezGonzalez procurou investigar se o controle condicional (diretamente ensinado e emergente) poderia ser exercido pela natureza das relações entre os componentes de estímulos modelo complexos. Numa fase inicial, foram treinadas as relações $\mathrm{AB}$ (A1B1, A2B2 e A3B3) e PQ (P1Q1, P2Q2 e P3Q3). Na sequência, no treino das relações $A B X$, as possíveis combinações dos estímulos A e B (Ex.: A1B1, A1B2, A1B3, $\mathrm{A} 2 \mathrm{~B} 1$, ...) foram apresentadas como estímulo modelo e os estímulos X1 e X2, como estímulos de escolha. A seleção do estímulo X1 foi reforçada apenas diante de um estímulo modelo definido pela relação modelo-escolha correta (ou seja: A1B1, A2B2 ou A3B3), enquanto que a seleção do estímulo X2 foi reforçada apenas diante do estímulo modelo definido pela relação modelo-escolha incorreta (ou seja: A1B2, A2B3, A3B1, A1B3, A2B1, A3B2). Em seguida, os testes das relações PQX avaliaram a transferência (ou extensão) das funções de controle das relações modeloescolha correta e modelo-escolha incorreta sobre a seleção dos estímulos X1 e X2. Dois dentre os quatro adultos alfabetizados e com desenvolvimento típico que participaram obtiveram resultados positivos sem a necessidade da exposição a procedimentos de treino adicionais (EF e EFX) e aos testes das relações simétricas BA e QP. No Experimento 2, os testes das relações simétricas BA e QP foram efetuados imediatamente após o treino das relações

\footnotetext{
${ }^{5}$ Este segundo tipo de delineamento admite uma interpretação distinta, a saber, em termos de uma unidade de análise de cinco termos (Lopes Jr \& Matos, 1995; Sidman, 1986, 1994), na qual um dos componentes do estímulo modelo exerce controle condicional sobre a função condiciona do outro componente, caracterizando, portanto, um controle condicional de segunda ordem ou controle contextual. Embora a discussão deste tópico não pertença ao escopo do presente trabalho, vale mencionar o registro de discussões pertinentes acerca destes modos de interpretação das funções discriminativas dos componentes do estímulo modelo complexo (Carpentier \& cols., 2000; Lynch \& Green, 1991; Markham \& Dougher, 1993; Sidman, 1994).
} 
$\mathrm{AB}$ e PQ, respectivamente, e precederam aos testes das relações PQX. Desta feita, todos os sujeitos (três adultos e um adolescente com desenvolvimento típico) atestaram a aprendizagem das relações treinadas, bem como a emergência das funções de controle avaliadas. Do Experimento 3 participaram os dois sujeitos do Experimento 1 que obtiveram resultados positivos nos testes de transferência e o adolescente do Experimento 2. De início, houve o treino das relações EFX: a seleção do estímulo X1 foi reforçada diante dos estímulos E1F1, E2F2 e E3F3; diante das demais combinações entre os estímulos E e F, a seleção do estímulo X2 foi reforçada. Em seguida, houve o teste das relações EF: os estímulos E1, E2 e E3 foram exibidos, sucessivamente, como estímulos modelo diante da apresentação simultânea dos estímulos de escolha F1, F2 e F3. Todos os sujeitos obtiveram resultados positivos nos testes, ou seja, estabeleceram relação de condicionalidade entre os estímulos diante dos quais a seleção do estímulo X1 fora previamente reforçada. Em seu conjunto, os resultados de Perez-Gonzalez (1994) apontam que a função de controle condicional não foi exercida apenas 1) pela identificação dos componentes do estímulo modelo, mas também 2) pela discriminação da natureza da relação sustentada entre tais componentes: manterem ou não condicionalidade prévia e diretamente ensinada. Em termos de aprendizagem discriminativa, os resultados de Perez-Gonzalez sugerem que a ocorrência da primeira (1) constitui-se em condição suficiente para a manifestação da segunda (2).

Lopes Jr., Costa, Gonsales e Golfeto (2001) avaliaram se a aprendizagem relacional atestada pelos participantes do estudo de Perez-Gonzalez seria igualmente registrada com crianças de menor faixa etária, apresentando ou não atraso de desenvolvimento intelectual. Utilizando desenhos não representacionais, no Experimento 1 cinco crianças de sala de ensino especial foram expostas ao treino de duas relações condicionais AB (A1B1 e A2B2) e aos testes das respectivas relações simétricas. Em seguida, ocorreu o treino de quatro relações ABX: a seleção do estímulo X1 seria correta diante dos estímulos modelos A1B1 e A2B2; diante dos estímulos modelos A1B2 e A2B1, a seleção do estímulo X2 foi definida como correta. Finalizado o treino das relações $\mathrm{ABX}$, os alunos foram expostos ao treino das relações condicionais PQ (P1Q1 e P2Q2) e aos testes das respectivas relações simétricas QP. Por fim, o teste das relações PQX avaliou se as relações entre os estímulos P e $\mathrm{Q}$, componentes do estímulo modelo, exerceriam controle condicional sobre a seleção dos estímulos X1 e X2. Apenas um dentre os cinco alunos atestou resultados positivos em todos os treinos e testes realizados. Para os quatro alunos restantes verificou-se um dado consistente: independente da emergência ou não das relações simétricas $\mathrm{BA}$, todos registraram resultados negativos no treino das relações $\mathrm{ABX}$. A questão de pesquisa, portanto, seria: como estabelecer, através de treino, o controle condicional pela natureza das relações entre os componentes do estímulo modelo complexo sobre a seleção de estímulos de escolha? Lopes Jr. e colaboradores (2001), no Experimento 2, avaliaram a seguinte hipótese: o insucesso registrado poderia ser eliminado se o treino das relações $\mathrm{ABX}$ fosse precedido pelo treino e teste de outras relações condicionais com estímulos modelos complexos, nos quais os participantes deveriam igualmente aprender a responder a relações entre os componentes do estímulo modelo? Em outros termos, o treino das relações $\mathrm{ABX}$ deveria ser precedido por contingências (treino e teste) que fornecessem instruções sobre as exigências discriminativas do treino ABX. Assim, precedendo a exposição aos treinos das relações $\mathrm{ABX}$ envolvendo relações de condicionalidade (A1B1 e A2B2) e de nãocondicionalidade (A1B2 e A2B1) com estímulos não representacionais, duas crianças de sala especial e duas crianças da segunda série do ensino fundamental, sem experiência prévia de participação no experimento, foram expostas ao treino de duas relações condicionais de identidade (F1F1 e F2F2), também com estímulos não-representacionais. Em seguida, o treino de quatro relações condicionais FFX determinava que, se os componentes do estímulo modelo sustentassem relações de identidade (F1F1 e F2F2), a seleção do estímulo X1 seria reforçada; em oposição, o estímulo $\mathrm{X} 2$ deveria ser selecionado se os componentes do estímulo modelo não sustentassem relações de identidade (F1F2 e F2F1). Treinos posteriores estabeleceram duas novas relações condicionais de identidade (Z1Z1 e Z2Z2). Finalizando a Fase 1, testes das relações ZZX avaliaram se a existência de relações de identidade e de não-identidade entre os componentes do estímulo modelo complexo (ZZ) exerceria controle sobre a seleção dos estímulos X1 e X2. A Fase 2 replicou o Experimento 1. As quatro crianças demonstraram a aprendizagem das relações FFX, bem como a emergência das relações ZZX. Uma criança de sala de ensino especial e outra do ensino fundamental igualmente registraram o critério de aprendizagem no treino das relações $\mathrm{ABX}$ e a emergência das relações PQX na Fase 2. As duas restantes registraram insucesso no treino das relações ABX. Portanto, em Lopes Jr. e colaboradores (2001), dentre nove crianças, três replicaram desempenhos previamente registrados na literatura sobre aprendizagem relacional por Perez-Gonzalez (1994). Seis crianças, por sua vez, demonstraram que a aprendizagem das relações $\mathrm{ABX}$ reserva dificuldades que deveriam ser alvo de investigações mais detalhadas.

$\mathrm{O}$ insucesso registrado na aprendizagem das relações ABX impõe uma questão preliminar: as dificuldades estariam 
funcionalmente relacionadas com a utilização de estímulos modelos complexos ou, diferentemente, com a natureza das relações entre os componentes dos estímulos modelos complexos? O treino das relações ABX exige, dentre outras habilidades, que o aprendiz responda, simultâneamente, aos dois componentes do estímulo modelo. Dificuldades no estabelecimento do controle discriminativo com estímulos complexos constituem-se em dados recorrentes da literatura, sendo que uma parcela das medidas comportamentais registradas documenta o fenômeno do controle restrito de estímulos, seletividade perceptual, atenção seletiva ou seletividade de estímulo (Burke, 1991; Dube \& McIlvane, 1997, 1999; Huguenin \& Touchette, 1980; Koegel \& Schreibman, 1977; Litrownik, McInnis, Wetzel-Pritchard \& Filipelli, 1978; Lovaas, Koegel \& Schreibman, 1979; Lovaas, Schreibman, Koegel \& Rehm, 1971; McIlvane, Dube \& Callahan, 1996; Reynolds, 1961; Schreibman, Charlop \& Koegel, 1982; R. Stromer \& cols., 1993a).

Dube e McIlvane (1999) forneceram uma ilustrativa demonstração das dificuldades associadas com o estabelecimento do controle condicional com estímulos modelos complexos. Três adolescentes com diagnóstico de deficiência mental foram expostos inicialmente (Fase 1) ao treino de relações condicionais através do matching-to-sample com atraso. Estímulos modelos complexos constituídos por dois desenhos arbitrários não-representacionais (Ex.: A1B1) foram expostos no centro da tela de um monitor. Um toque nos desenhos produzia o desaparecimento dos mesmos e a exibição de três estímulos unitários, um em cada vértice da tela (Ex.: A1, B2 e C1). Destes, apenas um sustentava relação de identidade com um dos componentes do estímulo modelo complexo. A seleção deste estímulo produzia reforço. A Fase 1 consistiu no treino de relações condicionais de identidade parcial. Na Fase 2, Dube e McIlvane (1999) introduziram o procedimento de resposta de observação diferencial (DOR-Differential Observing Response, p. 26). A resposta de pressionar o estímulo modelo complexo (Ex.: A1B1) na tela produzia três pares de estímulos de escolha, um em cada vértice, com manutenção do estímulo modelo. Destes três pares, um sustentava relação de identidade física com o modelo (A1B1) e nos demais, apenas um dos estímulos do par sustentava tal relação (Ex.: A1B2 e A2B1). A tarefa do participante consistia em estabelecer um matching-to-sample de identidade simultâneo com estímulos (modelo e escolha) complexos (A1B1-A1B1); em outros termos, o participante deveria fornecer uma medida comportamental de um responder sob controle dos dois componentes do estímulo modelo. Segundo Dube e McIlvane (1999), a utilização das respostas de observação diferenciais pode estabelecer condições eficientes que favorecem a discriminação dos componentes de estímulos complexos. Independente do estabelecimento do matchingto-sample de identidade com estímulos complexos, imediatamente após a seleção de um dos estímulos de escolha, estes foram retirados da tela, com manutenção do mesmo estímulo modelo complexo. Um novo toque neste estímulo produzia, desta feita, estímulos de escolha unitários nos vértices, sendo que apenas um era idêntico a um dos componentes do estímulo modelo. Portanto, na Fase 2, o treino de relações condicionais de identidade parcial foi precedido pela exposição ao matching-to-sample de identidade com estímulos (modelo e escolha) complexos. Por fim, a Fase 3 foi idêntica à Fase 1, ou seja, o estabelecimento do matching-to-sample de identidade parcial com estímulo modelo complexo não foi mais precedido pelo matching-to-sample de identidade com estímulos complexos. $\mathrm{Na}$ Fase 1, os dados dos três participantes oscilaram em níveis de acaso, sugerindo fortemente a ocorrência do controle restrito de estímulo, ou seja, a seleção dos estímulos de escolha foi controlada por apenas parte do estímulo modelo. $\mathrm{Na}$ Fase 2, elevados percentuais de acerto no estabelecimento do matching-to-sample de identidade com estímulos complexos foram acompanhados por significativa melhora na seleção do estímulo de escolha unitário que sustentava relação de identidade com um dos componentes do estímulo modelo complexo. $\mathrm{Na}$ Fase 3, com a retirada da exigência das respostas de observação diferenciais, ou seja, do estabelecimento do matching-to-sample de identidade com estímulos (modelo e escolha) complexos, para os três participantes, os índices de acerto retornaram aos níveis de acaso, a exemplo da Fase 1. Dube e McIlvane (1999) salientaram que, para os três participantes, o estabelecimento do matching-to-sample de identidade com estímulos complexos na Fase 2 mostrou-se eficiente na eliminação do controle restrito de estímulo, todavia com a retirada de uma condição que exigia um responder aos componentes do estímulo complexo (modelo e escolha), observou-se a reincidência do controle restrito de estímulo. Assim, a mera exposição a procedimentos que eliminam a ocorrência do controle restrito de estímulo não se mostrou suficientemente eficaz para manter o controle por todos os componentes do estímulo complexo na ausência destes procedimentos.

A questão básica do presente estudo consistiu em avaliar, com crianças de sala de ensino especial, quais efeitos poderiam ser observados na aprendizagem das relações condicionais $\mathrm{ABX}$ e na extensão desta modalidade de controle quando a exposição aos treinos e testes fosse precedida por contingências de treino que exigissem respostas de observação diferenciais (DOR), na acepção de Dube e McIlvane (1999), ou seja, a exposição ao matchingto-sample de identidade com estímulos (modelo e escolha) complexos. O procedimento adotado por Dube e McIlvane 
na Fase 2 viabiliza uma análise de desempenho sob distintas condições de aprendizagem. Teríamos, portanto, as seguintes questões derivadas: 1) insucessos na aprendizagem das relações ABX irão suceder insucessos no estabelecimento do matching-to-sample de identidade com estímulos complexos, sugerindo fortemente que, a despeito da complexidade das relações que definem quando X1 e X2 serão S+ ou S- diante dos estímulos A e B, os sujeitos evidenciam dificuldades com a própria observação dos dois componentes do estímulo modelo; 2) estabelecer o matching-to-sample de identidade com estímulos complexos pode não garantir a aprendizagem posterior das relações $\mathrm{ABX}$, sugerindo que possivelmente não se trate de controle restrito de estímulo; e, finalmente, 3) sucesso em ambos sugeriria que a exposição prévia ao matching-to-sample de identidade com estímulos complexos foi eficiente o bastante para eliminar fontes de controle de estímulo que dificultavam a aprendizagem das relações $\mathrm{ABX}$; deste modo, a questão adicional seria: 4) a obtenção do critério de aprendizagem no treino das relações ABX seria suficiente para demonstração da extensão desta modalidade de controle condicional para outras relações (Ex.: PQX)?

Tabela 1

Características dos Participantes do Experimento características do estudo, em termos das dificuldades de aprendizagem demonstradas em sala de aula. A autorização para participação no estudo foi obtida pela coordenação pedagógica em reunião com os pais ou responsáveis pelos alunos indicados.

\section{Material}

Todas as sessões de coleta de dados foram realizadas na própria escola dos participantes, em sala (3,5 m x 5,0 m) devidamente reservada para esta finalidade, com condições adequadas de isolamento acústico e de luminosidade.

Nesta sala, sobre uma carteira escolar idêntica àquelas utilizadas pelos participantes na sala de aula, foi instalado um microcomputador (PC) com monitor (15 polegadas) colorido. Um software, desenvolvido especificamente para este projeto, controlou a exibição dos estímulos na tela do monitor e efetuou o registro e a gravação em arquivo de dados das respostas de seleção dos estímulos através do mouse. Os estímulos foram quatro desenhos representacionais coloridos - limão (R1), banana (R2), boi (R3) e cavalo (R4) - retirados, através de scanner, de cartilhas escolares, bem como dez desenhos não representacionais, ocupando, cada desenho, uma área de $2,5 \mathrm{~cm} \mathrm{x} \mathrm{2,5} \mathrm{cm,}$

\begin{tabular}{ccccccc}
\hline Participante & Sexo & Idade (ano/mês) & QI execução & QI verbal & QI total & Informação diagnóstica* \\
\hline 1 & $\mathrm{M}$ & $12 / 2$ & 82 & 60 & 67 & Deficiente mental \\
2 & $\mathrm{M}$ & $9 / 7$ & 82 & 81 & 80 & Médio inferior \\
3 & $\mathrm{M}$ & $9 / 11$ & 89 & 85 & 85 & Médio inferior
\end{tabular}

Nota. * WISC - Escala Weschler de Inteligência para crianças, aplicada antes do início da coleta de dados.

\section{Método}

\section{Participantes}

Participaram três alunos regularmente matriculados em sala de ensino especial de uma escola da rede pública estadual que atende a uma população de nível sócioeconômico baixo. Para esses alunos a participação nesse estudo constituiu-se na primeira experiência com tarefas de matching-to-sample envolvendo recursos de informática em pesquisa sobre controle de estímulo. As principais informações de caracterização dos participantes são exibidas na Tabela 1.

A indicação dos participantes foi realizada pela coordenação pedagógica da escola e pela professora da sala de ensino especial após reunião com os pesquisadores na qual foram apresentados os objetivos do trabalho, com uma descrição dos procedimentos e dos recursos que deveriam ser utilizados. Foram indicados para participação aqueles alunos que poderiam ser mais beneficiados, pelas exibidos com traçado preto em fundo branco. A Figura 1 indica os desenhos não representacionais com a respectiva designação alfa-numérica.

Esta designação foi utilizada apenas para efeito de referência neste texto, sendo que aos participantes foram exibidos apenas os desenhos.

\section{Procedimento Geral}

As sessões experimentais foram realizadas três vezes por semana, no horário regular de aula dos participantes. Durante cada sessão permaneciam na sala apenas o participante e o experimentador. A duração média de cada sessão foi de 25 minutos.

O software utilizado permitiu a exibição de três tipos distintos de tentativas. Nas tentativas do Tipo 1, a tela foi iniciada com um estímulo (representacional ou não) exibido numa janela retangular $(3,5 \mathrm{~cm}$ x 4,0 cm) disposta na posição central. Uma pressão no botão esquerdo do 


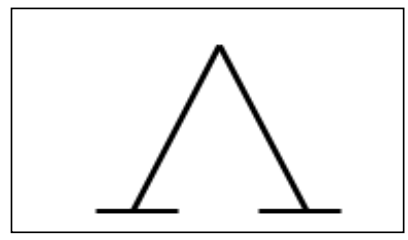

A1

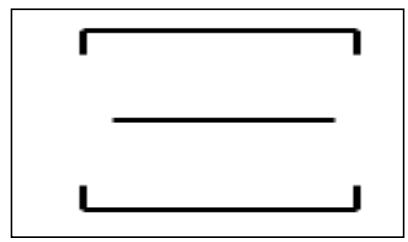

B1

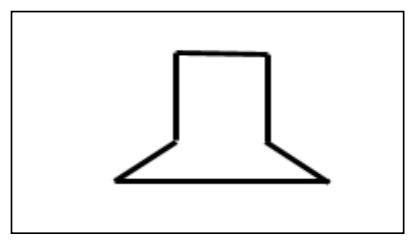

X1

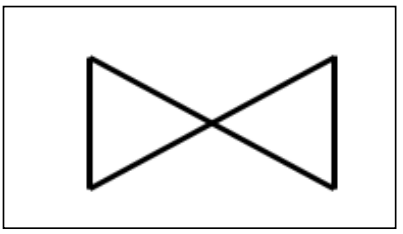

P1

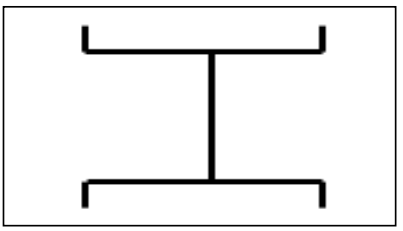

Q1

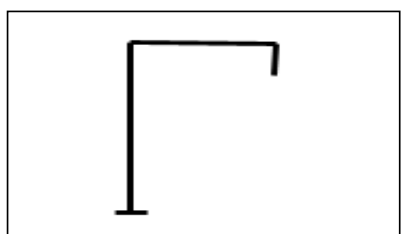

A2

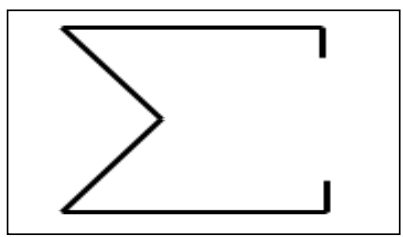

B2

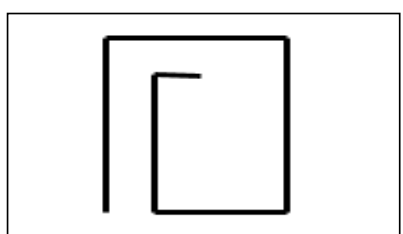

X2

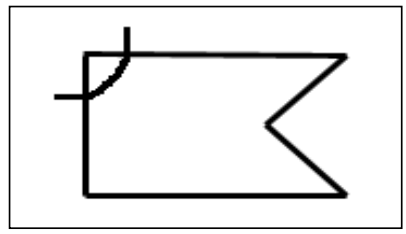

P2

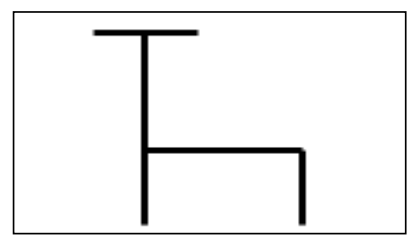

Q2
Figura 1. Estímulos arbitrários não-representacionais utilizados no Experimento 1. A designação alfanumérica é utilizada para facilitar a referência aos mesmos no texto. Aos participantes foram exibidos apenas os respectivos desenhos.

mouse com o cursor sobre esse estímulo central produzia quatro janelas com o mesmo tamanho da anterior, sendo uma em cada vértice da tela, com a manutenção do estímulo exibido na posição central. Dois estímulos de comparação foram apresentados simultaneamente nessas janelas periféricas, sendo que a cada tentativa duas janelas permaneciam em branco (sem exibição de desenho). Recursos de randomização do software definiram as posições dos estímulos de escolha, bem como as janelas que foram exibidas em branco. A resposta de escolha consistiu na colocação do cursor sobre um dos estímulos exibidos nas janelas periféricas e um click no botão esquerdo do mouse. A emissão da resposta de escolha produzia uma, dentre duas telas de feedback: uma tela de acerto que consistia na exibição de uma imagem indicativa de sucesso (Ex.: sinal de positivo, um aperto de mão) e uma tela de erro (Ex.: um sinal de negativo). A tela de feedback foi exibida por dois segundos, sendo sucedida por uma tela escura (ITI) com duração de 1,5 segundos, após a qual iniciava-se uma nova tentativa. A telas de feedback foram utilizadas com os demais tipos de tentativas quando o procedimento previa o reforçamento diferencial. Quando o procedimento não estabelecia reforçamento diferencial, a seleção do estímulo de escolha produzia a tela escura (ITI), iniciando-se, em seguida uma nova tentativa. A tentativa do Tipo 1 foi utilizada nos treinos e nos testes das relações condicionais especificadas com duas letras (Ex. AB, BA). Nestes casos, a primeira letra (da esquerda para a direita) indica o conjunto dos estímulos que foram exibidos na posição central (Ex.: para as relações $A B$, estímulos do conjunto $A$ : $A 1$ ou A2), enquanto que a segunda letra indica o conjunto dos estímulos de escolha exibidos nos vértices (dado o exemplo, os estímulos B1 e B2).

$\mathrm{Na}$ tentativa do Tipo 2, a tela foi iniciada com dois estímulos exibidos simultaneamente, um ao lado do outro, numa janela central (estímulo modelo complexo) ocupando uma área de $3,5 \mathrm{~cm}$ x $8 \mathrm{~cm}$. Desta feita, uma resposta de pressão no botão esquerdo do mouse com o cursor colocado sobre a área de projeção do estímulo modelo produzia a exibição dos estímulos de escolha nas janelas periféricas. Cada estímulo de escolha consistiu num par de estímulos exibidos simultaneamente e lado a lado, em áreas de $3,5 \mathrm{~cm}$ x $8 \mathrm{~cm}$ nos vértices da tela. A cada tentativa, o click no mouse com o cursor na posição central produzia a exibição de três pares de desenhos, sendo cada par localizado num dos vértices, com manutenção do estímulo condicional complexo na posição central. As tentativas do Tipo 2 foram utilizadas nos treinos das relações condicionais de identidade com estímulos (modelo e de escolha) complexos e designadas pela notação $A B-A B$, na qual o primeiro par especifica o conjunto dos componentes do estímulo modelo complexo e o segundo par, os componentes do estímulo de escolha complexo. Na tentativa do Tipo 2, a seleção do estímulo de escolha incorreto produzia a exibição da tela de erro (em caso de reforçamento diferencial) ou diretamente a tela escura (ITI), com início posterior da tentativa seguinte. Diferentemente, a seleção do estímulo de escolha correto, ou seja, o estabelecimento do matching- 
to-sample de identidade com estímulos complexos, produzia o início do terceiro tipo de tentativa (Tipo 3).

As tentativas de Tipo 3 foram iniciadas com a manutenção do estímulo modelo complexo da tentativa Tipo 2 imediatamente anterior no centro da tela, sem qualquer estímulo nos vértices da tela. Uma resposta de pressão no mouse com o cursor localizado sobre a área de projeção do estímulo modelo complexo produzia as mesmas conseqüências previamente descritas para a resposta no estímulo central na tentativa do Tipo 1 , desta feita, com a manutenção do estímulo modelo complexo no centro da tela. De modo similar, as respostas de seleção dos estímulos de escolha produziam ou tela de feedback ou a tela escura (ITI). As tentativas do Tipo 3 foram utilizadas nos treinos e nos testes de relações condicionais especificados com três letras, como por exemplo, ABX, sendo que as duas letras iniciais (da esquerda para a direita) informam os conjuntos dos estímulos modelo e a terceira letra, o conjunto dos estímulos de escolha. As tentativas dos Tipos 2 e 3 foram ilustradas em Dube e McIlvane (1999, Fig.1, p. 27).
O procedimento previa a execução da avaliação psicométrica dos alunos encaminhados, seguida de um pré-treino e de duas fases, sendo cada uma composta pelo treino e teste de relações condicionais.

Pré-treino- De início, os participantes foram expostos às tentativas do Tipo 1, com desenhos representacionais, com o objetivo de familiarizá-los com a rotina de sessões de coleta de dados, com o ambiente de coleta, com a operação do mouse e a seleção dos desenhos na tela. Todos os participantes, na primeira sessão, foram informados de que se tratava de um jogo e que, para vencê-lo, era necessário acertar o máximo possível. Diante a tentativa inicial (Tipo 1), o experimentador dizia que o participante deveria trabalhar com o mouse e descobrir como acertar os exercícios do jogo. Nas duas tentativas iniciais, o experimentador descreveu verbalmente o modo de operação do mouse, a seleção dos estímulos na tela e o significado das telas de feedback, sendo que o próprio participante emitia as respostas motoras de manipulação do mouse. A Tabela 2 descreve a composição dos blocos de tentativas expostos aos participantes, o respectivo número

Tabela 2

Composição dos Blocos e Seqüência de Apresentação dos mesmos no Pré-treino e na Fase 1, com os Respectivos Critérios de Reforçamento

\begin{tabular}{|c|c|c|c|c|c|c|c|c|}
\hline \multicolumn{6}{|c|}{ Relações condicionais } & \multirow{2}{*}{$\begin{array}{l}\text { Critério de } \\
\text { reforcamento }\end{array}$} & \multirow{2}{*}{$\begin{array}{c}\text { Bloco/Acesso } \\
\text { (Acerto) }\end{array}$} & \multirow{2}{*}{$\begin{array}{c}\text { Bloco/Acesso } \\
\text { (Erro) }\end{array}$} \\
\hline Fase & Bloco & Modelo & $\mathrm{S}+$ & $\mathrm{S}-$ & $\mathrm{N}^{\circ}$ de tentativas & & & \\
\hline \multirow{7}{*}{$\begin{array}{l}\text { Pré } \\
\text { Treino }\end{array}$} & 1 & R1 & $\mathrm{R} 2$ & $\mathrm{R} 4$ & 4 & $\mathrm{RD}^{\mathrm{a}}$ & 2 & 1 \\
\hline & 2 & R3 & R4 & $\mathrm{R} 2$ & 4 & RD & 3 & 1 \\
\hline & 3 & R1 & $\mathrm{R} 2$ & $\mathrm{R} 4$ & 2 & RD & 4 & 1 \\
\hline & & R3 & $\mathrm{R} 4$ & $\mathrm{R} 2$ & 2 & & & \\
\hline & 4 & R1 & $\mathrm{R} 2$ & $\mathrm{R} 4$ & 2 & RD & 5 & 3 \\
\hline & & R3 & $\mathrm{R} 4$ & $\mathrm{R} 2$ & 2 & & & \\
\hline & 5 & R1 & $\mathrm{R} 2$ & B1 & 2 & $\mathrm{RD}$ & 6 & 4 \\
\hline Fase 1 & 6 & A1 & B1 & $\mathrm{R} 2$ & 2 & $\mathrm{RD}$ & 7 & 4 \\
\hline \multirow{5}{*}{$\begin{array}{c}\text { Treino } \\
\text { AB }\end{array}$} & 7 & R1 & $\mathrm{R} 2$ & B1 & 2 & RD & 8 & 4 \\
\hline & & A1 & B1 & $\mathrm{R} 2$ & 2 & & & \\
\hline & 8 & A1 & B1 & B2 & 1 & RD & 9 & 7 \\
\hline & 9 & A2 & B2 & B1 & 1 & $\mathrm{RD}$ & 10 & 7 \\
\hline & 10 & A1 & B1 & B2 & 3 & RD & 11 & 7 \\
\hline \multirow{7}{*}{$\begin{array}{c}\text { Fase } 1 \\
\text { Teste } \\
\text { BA }\end{array}$} & & A2 & B2 & B1 & 3 & & & \\
\hline & 11 & A1 & B1 & B2 & 3 & $\mathrm{EXT}^{\mathrm{b}}$ & 12 & 10 \\
\hline & & A2 & B2 & B1 & 3 & & & \\
\hline & 12 & B1 & A1 & A2 & 5 & $\mathrm{SCP}^{\mathrm{c}}$ & 13 & 13 \\
\hline & & B2 & A 2 & A1 & 5 & & & \\
\hline & 13 & A1 & B1 & B2 & 2 & $\mathrm{RD}$ & FIM $^{\mathrm{d}}$ & FIM \\
\hline & & A2 & B2 & B1 & 2 & & & \\
\hline
\end{tabular}

${ }^{\mathrm{a}} \mathrm{RD}$ - Reforçamento Diferencial

${ }^{\mathrm{b}}$ EXT - Extinção

c SCP - Sem Conseqüência Programada

${ }^{\mathrm{d}}$ FIM - Encerramento da exibição dos estímulos com fechamento automático do programa.

Esta legenda foi adotada nas Tabelas 3 e 4.

Psicologia: Reflexão e Crítica, 2003, 16(1), pp. 71-84 
Tabela 3

Composição dos Blocos e Sequiencia de Apresentação dos mesmos no Treino das Relações $A B-A B$ e ABX, com os Respectivos Critérios de Reforçamento

\begin{tabular}{|c|c|c|c|c|c|c|c|}
\hline \multirow[b]{2}{*}{ BLOCO } & \multicolumn{3}{|c|}{ Relações condicionais } & \multirow{2}{*}{$\begin{array}{c}\text { Número } \\
\text { deTentativas }\end{array}$} & \multirow{2}{*}{$\begin{array}{c}\text { Critério de } \\
\text { reforçamento }\end{array}$} & \multirow{2}{*}{$\begin{array}{c}\text { Bloco/Acesso } \\
\text { (Acerto) }\end{array}$} & \multirow{2}{*}{$\begin{array}{c}\text { Bloco/Acesso } \\
\text { (Erro) }\end{array}$} \\
\hline & Modelo & $\mathrm{S}+$ & $\mathrm{S}-$ & & & & \\
\hline \multirow[t]{3}{*}{1} & A1 & B1 & $\mathrm{B} 2$ & 4 & $\mathrm{RD}$ & 3 & 2 \\
\hline & A2 & B2 & B1 & & & & \\
\hline & $\mathrm{A} 1$ & $\mathrm{~B} 1$ & $\mathrm{~B} 2$ & 4 & $\mathrm{RD}$ & 3 & FIM \\
\hline \multirow[t]{6}{*}{2} & $\mathrm{~A} 2$ & $\mathrm{~B} 2$ & B1 & & & & \\
\hline & A1 & B1 & B2 & 2 & & & \\
\hline & A1B1 & A1B1 & A2B1 e A1B2 & 2 & & & \\
\hline & A1B1 & $\mathrm{X} 1$ & $\mathrm{X} 2$ & 2 & & & \\
\hline & A2 & B2 & B1 & 2 & & & \\
\hline & $\mathrm{A} 2 \mathrm{~B} 2$ & $\mathrm{~A} 2 \mathrm{~B} 2$ & $\mathrm{~A} 2 \mathrm{~B} 1$ e $\mathrm{A} 1 \mathrm{~B} 2$ & 2 & $\mathrm{RD}$ & 6 & 4 \\
\hline \multirow[t]{9}{*}{3} & $\mathrm{~A} 2 \mathrm{~B} 2$ & $\mathrm{X} 1$ & $\mathrm{X} 2$ & 2 & & & \\
\hline & A1B2 & $\mathrm{A} 1 \mathrm{~B} 2$ & A1B1 e A2B2 & 2 & & & \\
\hline & $\mathrm{A} 1 \mathrm{~B} 2$ & $\mathrm{X} 2$ & $\mathrm{X} 1$ & 2 & & & \\
\hline & $\mathrm{A} 2 \mathrm{~B} 1$ & $\mathrm{~A} 2 \mathrm{~B} 1$ & $\mathrm{~A} 2 \mathrm{~B} 2$ e $\mathrm{A} 1 \mathrm{~B} 1$ & 2 & & & \\
\hline & $\mathrm{A} 2 \mathrm{~B} 1$ & $\mathrm{X} 2$ & $\mathrm{X} 1$ & 2 & & & \\
\hline & A1 & B1 & B2 & 2 & & & \\
\hline & A1B1 & A1B1 & $\mathrm{A} 2 \mathrm{~B} 1$ e $\mathrm{A} 1 \mathrm{~B} 2$ & 2 & & & \\
\hline & A1B1 & X1 & $\mathrm{X} 2$ & 2 & & & \\
\hline & A2 & $\mathrm{B} 2$ & B1 & 2 & $\mathrm{RD}$ & 6 & 5 \\
\hline \multirow[t]{10}{*}{4} & $\mathrm{~A} 2 \mathrm{~B} 2$ & $\mathrm{~A} 2 \mathrm{~B} 2$ & A2B1 e A1B2 & 2 & & & \\
\hline & A2B2 & $\mathrm{X} 1$ & $\mathrm{X} 2$ & 2 & & & \\
\hline & $\mathrm{A} 1 \mathrm{~B} 2$ & $\mathrm{~A} 1 \mathrm{~B} 2$ & $\mathrm{~A} 1 \mathrm{~B} 1$ e $\mathrm{A} 2 \mathrm{~B} 2$ & 2 & & & \\
\hline & A1B2 & $\mathrm{X} 2$ & $\mathrm{X} 1$ & 2 & & & \\
\hline & $\mathrm{A} 2 \mathrm{~B} 1$ & $\mathrm{~A} 2 \mathrm{~B} 1$ & $\mathrm{~A} 2 \mathrm{~B} 2$ e $\mathrm{A} 1 \mathrm{~B} 1$ & 2 & & & \\
\hline & $\mathrm{A} 2 \mathrm{~B} 1$ & $\mathrm{X} 2$ & $\mathrm{X} 1$ & 2 & & & \\
\hline & A1 & B1 & B2 & 2 & & & \\
\hline & A1B1 & A1B1 & A2B1 e A1B2 & 2 & & & \\
\hline & A1B1 & $\mathrm{X} 1$ & X2 & 2 & & & \\
\hline & A2 & B2 & B1 & 2 & & & \\
\hline \multirow[t]{9}{*}{5} & A2B2 & $\mathrm{A} 2 \mathrm{~B} 2$ & A2B1 e A1B2 & 2 & $\mathrm{RD}$ & 6 & FIM \\
\hline & $\mathrm{A} 2 \mathrm{~B} 2$ & X1 & $\mathrm{X} 2$ & 2 & & & \\
\hline & A1B2 & $\mathrm{A} 1 \mathrm{~B} 2$ & $\mathrm{~A} 1 \mathrm{~B} 1$ e $\mathrm{A} 2 \mathrm{~B} 2$ & 2 & & & \\
\hline & A1B2 & $\mathrm{X} 2$ & $\mathrm{X} 1$ & 2 & & & \\
\hline & $\mathrm{A} 2 \mathrm{~B} 1$ & $\mathrm{~A} 2 \mathrm{~B} 1$ & $\mathrm{~A} 2 \mathrm{~B} 2$ e $\mathrm{A} 1 \mathrm{~B} 1$ & 2 & & & \\
\hline & $\mathrm{A} 2 \mathrm{~B} 1$ & $\mathrm{X} 2$ & $\mathrm{X} 1$ & 2 & & & \\
\hline & A1B1 & A1B1 & $\mathrm{A} 2 \mathrm{~B} 1$ e $\mathrm{A} 1 \mathrm{~B} 2$ & 2 & & & \\
\hline & A1B1 & $\mathrm{X} 1$ & $\mathrm{X} 2$ & 2 & & & \\
\hline & A2B2 & $\mathrm{A} 2 \mathrm{~B} 2$ & A2B1 e A1B2 & 2 & & & \\
\hline \multirow[t]{8}{*}{6} & A2B2 & $\mathrm{X} 1$ & $\mathrm{X} 2$ & 2 & & & \\
\hline & $\mathrm{A} 1 \mathrm{~B} 2$ & $\mathrm{~A} 1 \mathrm{~B} 2$ & A1B1 e A2B2 & 2 & $\mathrm{RD}$ & FIM & 7 \\
\hline & A1B2 & $\mathrm{X} 2$ & $\mathrm{X} 1$ & 2 & & & \\
\hline & $\mathrm{A} 2 \mathrm{~B} 1$ & $\mathrm{~A} 2 \mathrm{~B} 1$ & $\mathrm{~A} 2 \mathrm{~B} 2$ e $\mathrm{A} 1 \mathrm{~B} 1$ & 2 & & & \\
\hline & $\mathrm{A} 2 \mathrm{~B} 1$ & $\mathrm{X} 2$ & $\mathrm{X} 1$ & 2 & & & \\
\hline & A1B1 & A1B1 & $\mathrm{A} 2 \mathrm{~B} 1$ e $\mathrm{A} 1 \mathrm{~B} 2$ & 2 & & & \\
\hline & A1B1 & $\mathrm{X} 1$ & $\mathrm{X} 2$ & 2 & $\mathrm{RD}$ & FIM & 8 \\
\hline & A2B2 & $\mathrm{A} 2 \mathrm{~B} 2$ & A2B1 e A1B2 & 2 & & & \\
\hline \multirow[t]{8}{*}{7} & $\mathrm{~A} 2 \mathrm{~B} 2$ & X1 & $\mathrm{X} 2$ & 2 & & & \\
\hline & $\mathrm{A} 1 \mathrm{~B} 2$ & $\mathrm{~A} 1 \mathrm{~B} 2$ & A1B1 e A2B2 & 2 & & & \\
\hline & A1B2 & X2 & $\mathrm{X} 1$ & 2 & & & \\
\hline & $\mathrm{A} 2 \mathrm{~B} 1$ & $\mathrm{~A} 2 \mathrm{~B} 1$ & $\mathrm{~A} 2 \mathrm{~B} 2$ e $\mathrm{A} 1 \mathrm{~B} 1$ & 2 & & & \\
\hline & $\mathrm{A} 2 \mathrm{~B} 1$ & $\mathrm{X} 2$ & $\mathrm{X} 1$ & 2 & & & \\
\hline & A1B1 & A1B1 & $\mathrm{A} 2 \mathrm{~B} 1$ e $\mathrm{A} 1 \mathrm{~B} 2$ & 2 & & & \\
\hline & A1B1 & $\mathrm{X} 1$ & X2 & 2 & $\mathrm{RD}$ & FIM & FIM \\
\hline & A2B2 & $\mathrm{A} 2 \mathrm{~B} 2$ & $\mathrm{~A} 2 \mathrm{~B} 1$ e $\mathrm{A} 1 \mathrm{~B} 2$ & 2 & & & \\
\hline \multirow[t]{5}{*}{8} & $\mathrm{~A} 2 \mathrm{~B} 2$ & $\mathrm{X} 1$ & $\mathrm{x} 2$ & 2 & & & \\
\hline & $\mathrm{A} 1 \mathrm{~B} 2$ & $\mathrm{~A} 1 \mathrm{~B} 2$ & $\mathrm{~A} 1 \mathrm{~B} 1 \mathrm{e}$ A2B2 & 2 & & & \\
\hline & A1B2 & $\mathrm{X} 2$ & $\mathrm{X} 1$ & 2 & & & \\
\hline & $\mathrm{A} 2 \mathrm{~B} 1$ & $\mathrm{~A} 2 \mathrm{~B} 1$ & $\mathrm{~A} 2 \mathrm{~B} 2$ e $\mathrm{A} 1 \mathrm{~B} 1$ & 2 & & & \\
\hline & $\mathrm{A} 2 \mathrm{~B} 1$ & $\mathrm{X} 2$ & $\mathrm{X} 1$ & 2 & & & \\
\hline
\end{tabular}


de tentativas por bloco, bem como a seqüência determinada pelo software em função do desempenho (acerto ou erro) em cada bloco. O critério de aprendizagem adotado nos blocos com reforçamento diferencial (RD) e extinção (EXT) foi de $100 \%$ de acerto nas tentativas do bloco. Tal critério foi considerado nas demais fases do procedimento. A Tabela 2, na coluna Bloco/Acesso (Acerto), indica a seqüência e os blocos exibidos quando esse critério foi registrado; diferentemente, a coluna Bloco/Acesso (Erro) informa a seqüência e os blocos apresentados diante de insucessos na obtenção desse critério. No pré-treino, de acordo com o procedimento descrito na Tabela 2, foram treinadas duas relações condicionais: diante do estímulo modelo desenho do limão (R1) e dos estímulos de escolhas R2 e R4 (desenhos de banana e de cavalo, respectivamente) a seleção do desenho da banana (R2) produzia indicação de acerto; diante do modelo R3 (desenho do boi), desta feita, a seleção do desenho do cavalo (R4) foi considerada correta. A obtenção do critério de aprendizagem no Bloco 3 (Tabela 2) acarretava no acesso automático ao Bloco 4, iniciando o treino das relações condicionais $\mathrm{AB}$. Cinco insucessos no registro deste desempenho implicaria no encerramento da participação do aluno no experimento.

Fase 1 - Treino AB/Teste BA. De acordo com a indicação da Tabela 2, o treino das relações condicionais AB (A1B1 e A2B2) foi iniciado com uma revisão das duas relações treinadas no pré-treino (Bloco 4). Em seguida, observa-se a introdução do estímulo B1 como S- diante do modelo R1 (desenho do limão) e a introdução do A1 como estímulo modelo diante dos estímulos de escolha B1 e o desenho da banana (R2). Com o intuito de melhor caracterizar as relações de controle de estímulo estabelecidas pelas contingências de treino, no Bloco 8 diante do estímulo modelo A1 foram inseridos os estímulos de escolha B1 e B2 (primeira exibição). A obtenção do critério de aprendizagem no bloco que mesclava as duas relações $A B$ treinadas com reforçamento diferencial (Bloco 10) e em extinção (Bloco 11) produzia acesso automático ao bloco que exibia 10 tentativas de teste das relações simétricas BA (Bloco 12), sem conseqüência programada (SCP). Independente do desempenho neste bloco de teste, a Fase1 foi finalizada com nova exposição a um bloco de treino das duas relações AB (Bloco 13).

Fase 1 - Treino ABX. O procedimento adotado no treino das relações condicionais ABX é descrito na Tabela 3. Este procedimento previa uma revisão das relações condicionais $\mathrm{AB}$ (Blocos 1 e 2) e a exposição a blocos com exibição sucessiva das relações ABX precedida pelo treino das relações condicionais AB-AB. Por sua vez, este treino foi, ora precedido pela apresentação das relações $\mathrm{AB}$ (Blocos $4 \mathrm{e}$ 5), ora sem esta precedência (Blocos 6, 7 e 8). Nenhuma

Psicologia: Reflexão e Crítica, 2003, 16(1), pp. 71-84 instrução adicional foi fornecida para a interação dos participantes com estes diferentes tipos de tentativas, senão recordá-lo do objetivo de obter o maior número possível de acertos.

A Tabela 3 indica a seqüência adotada em função do desempenho registrado na exposição a esses blocos. A obtenção do critério de aprendizagem nos Blocos 6, 7 ou 8 acarretava no final da Fase 1. Com cinco insucessos no registro deste desempenho, a participação do aluno foi encerrada.

Fase 2 - Treino PQ/Teste QP. Na Fase 2 ocorreu a revisão das relações condicionais do pré-treino, seguida do treino das relações condicionais PQ (P1Q1 e P2Q2) e o teste das respectivas relações simétricas QP. Os procedimentos utilizados foram exatamente os mesmos descritos na Tabela 2, a partir do Bloco 4 (inclusive), para as relações $\mathrm{AB}$ e $\mathrm{BA}$, com as devidas adaptações.

Fase 3-Teste PQX. O teste das relações PQX avaliou se a natureza das relações entre os componentes do estímulo modelo (sustentar ou não condicionalidade previamente treinada) exerceria controle sobre a seleção dos estímulos X1 e X2. Deste modo, o teste das relações PQX foi precedido pela revisão das relações de linha de base (previamente treinadas) AB, ABX e PQ. A Tabela 4 descreve o procedimento adotado no teste das relações PQX, indicando a composição dos blocos apresentados em função do desempenho do participante (obtenção ou não do critério de aprendizagem).

De acordo com a previsão inicial, o término do experimento deveria ocorrer após a finalização do Bloco 7 (Tabela 4) independente do desempenho (número de acerto ou erros) neste bloco, ou após cinco insucessos na obtenção do critério de aprendizagem nos blocos que precederam o acesso ao Bloco 7.

\section{Resultados}

Os três participantes demonstraram as relações condicionais previstas no pré-treino com estímulos representacionais, bem como as duas relações condicionais com estímulos arbitrários não-representacionais $\mathrm{AB}$ (A1B1 e A2B2). No Bloco 8 (Tabela 2), do treino das relações AB, introduziu-se o estímulo de escolha incorreto (S-) B2. Esta medida objetivou avaliar qual a relação de controle de estímulo que estaria funcionalmente relacionada com a seleção do estímulo B1 precedendo ao Bloco 8: os participantes, diante do estímulo $\mathrm{A} 1$, rejeitavam $\mathrm{R} 2$ ou selecionavam $\mathrm{B} 1$ ? $\mathrm{Na}$ hipótese da rejeição do estímulo R2 podia-se prever desempenhos ao acaso a partir do Bloco 7, com exibição de dois estímulos de escolha desconhecidos (B1 e B2); diferentemente, a seleção de B1 diante do estímulo 
Tabela 4

Composição dos Blocos e Sequiência de Apresentação dos mesmos no Teste das Relações Condicionais PQX, com os Respectivos Critérios de Reforçamento

\begin{tabular}{|c|c|c|c|c|c|c|c|}
\hline \multirow[b]{2}{*}{ Bloco } & \multicolumn{3}{|c|}{ Relações condicionais } & \multirow{2}{*}{$\begin{array}{c}\text { Número } \\
\text { de tentativas }\end{array}$} & \multirow{2}{*}{$\begin{array}{l}\text { Critério de } \\
\text { reforçamento }\end{array}$} & \multirow{2}{*}{$\begin{array}{c}\text { Bloco/Acesso } \\
\text { (Acerto) }\end{array}$} & \multirow{2}{*}{$\begin{array}{c}\text { Bloco/Acesso } \\
\text { (Erro) }\end{array}$} \\
\hline & Modelo & $\mathrm{S}+$ & S- & & & & \\
\hline \multirow[t]{3}{*}{1} & A1 & B1 & B2 & 4 & $\mathrm{RD}$ & 3 & 2 \\
\hline & A2 & B2 & B1 & & & & \\
\hline & A1 & B1 & B2 & 4 & RD & 3 & FIM \\
\hline \multirow[t]{6}{*}{2} & A2 & B2 & B1 & & & & \\
\hline & A1 & B1 & B2 & 2 & & & \\
\hline & A1B1 & A1B1 & A2B1 e A1B2 & 2 & & & \\
\hline & A1B1 & $\mathrm{X} 1$ & $\mathrm{X} 2$ & 2 & & & \\
\hline & A2 & B2 & B1 & 2 & & & \\
\hline & $\mathrm{A} 2 \mathrm{~B} 2$ & $\mathrm{~A} 2 \mathrm{~B} 2$ & A2B1 e A1B2 & 2 & $\mathrm{RD}$ & 5 & 4 \\
\hline \multirow[t]{10}{*}{3} & $\mathrm{~A} 2 \mathrm{~B} 2$ & $\mathrm{X} 1$ & $\mathrm{X} 2$ & 2 & & & \\
\hline & A1B2 & $\mathrm{A} 1 \mathrm{~B} 2$ & A1B1 e A2B2 & 2 & & & \\
\hline & A1B2 & $\mathrm{X} 2$ & $\mathrm{X} 1$ & 2 & & & \\
\hline & A2B1 & A2B1 & $\mathrm{A} 2 \mathrm{~B} 2 \mathrm{e}$ A1B1 & 2 & & & \\
\hline & A2B1 & $\mathrm{X} 2$ & $\mathrm{X} 1$ & 2 & & & \\
\hline & A1 & B1 & B2 & 2 & & & \\
\hline & A1B1 & A1B1 & A2B1 e A1B2 & 2 & & & \\
\hline & A1B1 & $\mathrm{X} 1$ & $\mathrm{X} 2$ & 2 & & & \\
\hline & A2 & $\mathrm{B} 2$ & B1 & 2 & & & \\
\hline & $\mathrm{A} 2 \mathrm{~B} 2$ & A2B2 & A2B1 e A1B2 & 2 & $\mathrm{RD}$ & 5 & FIM \\
\hline \multirow[t]{6}{*}{4} & A2B2 & $\mathrm{X} 1$ & $\mathrm{X} 2$ & 2 & & & \\
\hline & A1B2 & $\mathrm{A} 1 \mathrm{~B} 2$ & $\mathrm{~A} 1 \mathrm{~B} 1$ e A2B2 & 2 & & & \\
\hline & $\mathrm{A} 1 \mathrm{~B} 2$ & X2 & X1 & 2 & & & \\
\hline & A2B1 & A2B1 & A2B2 e A1B1 & 2 & & & \\
\hline & A2B1 & X2 & $\mathrm{X} 1$ & 2 & & & \\
\hline & P1 & Q1 & Q2 & 2 & $\mathrm{RD}$ & 7 & 6 \\
\hline \multirow[t]{2}{*}{5} & P2 & Q2 & Q1 & 2 & & & \\
\hline & P1 & Q1 & Q2 & 2 & $\mathrm{RD}$ & 7 & FIM \\
\hline \multirow[t]{5}{*}{6} & P2 & Q2 & Q1 & 2 & & & \\
\hline & P1Q1 & P1Q1 & P1Q2 e P2Q1 & 3 & & & \\
\hline & P2Q2 & P2Q2 & P2Q1 e P1Q2 & 3 & & & \\
\hline & P1Q2 & P1Q2 & P1Q1 e P2Q2 & 3 & & & \\
\hline & P2Q1 & P2Q1 & P1Q1 e P2Q2 & 3 & & & \\
\hline \multirow[t]{4}{*}{7} & P1Q1 & $\mathrm{X} 1$ & $\mathrm{X} 2$ & 3 & SCP & FIM & FIM \\
\hline & P1Q2 & $\mathrm{X} 2$ & $\mathrm{X} 1$ & 3 & & & \\
\hline & P2Q1 & $\mathrm{X} 2$ & $\mathrm{X} 1$ & 3 & & & \\
\hline & $\mathrm{P} 2 \mathrm{Q} 2$ & $\mathrm{X} 1$ & $\mathrm{X} 2$ & 3 & & & \\
\hline
\end{tabular}

modelo A1 sugeriria o controle por seleção do S+. Todos os participantes, no Bloco 8, selecionaram o estímulo B1 e não o B2, diante do estímulo modelo A1.

Os participantes 1 e 2 necessitaram de uma única exposição ao treino das relações condicionais $\mathrm{AB}$ (Blocos 4 a 11 - Tabela 2) para o registro do critério de aprendizagem nesta fase, enquanto que para o Participante 3 foram necessárias duas exposições. A despeito destas diferenças, os três participantes atestaram a emergência imediata das relações simétricas (BA) testadas.

$\mathrm{Na}$ fase seguinte, juntamente com a revisão das relações condicionais $\mathrm{AB}$, ocorreram os treinos das relações condicionais de identidade com estímulos complexos (AB$A B)$ e das quatro relações $A B X$. A Figura 2 indica os percentuais de acerto registrados pelos participantes nas cinco exposições ao procedimento na Tabela 3. 

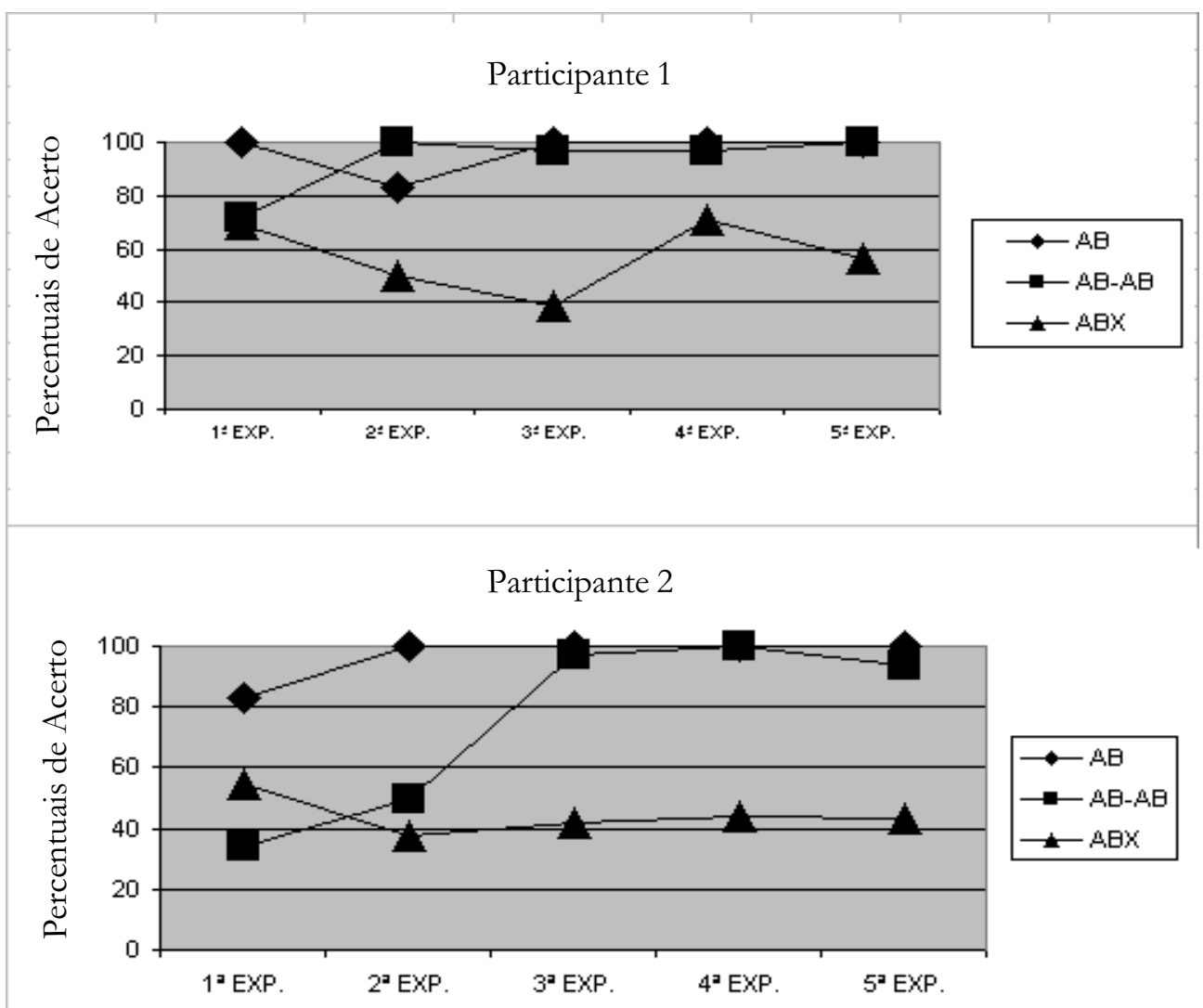

Participante 3

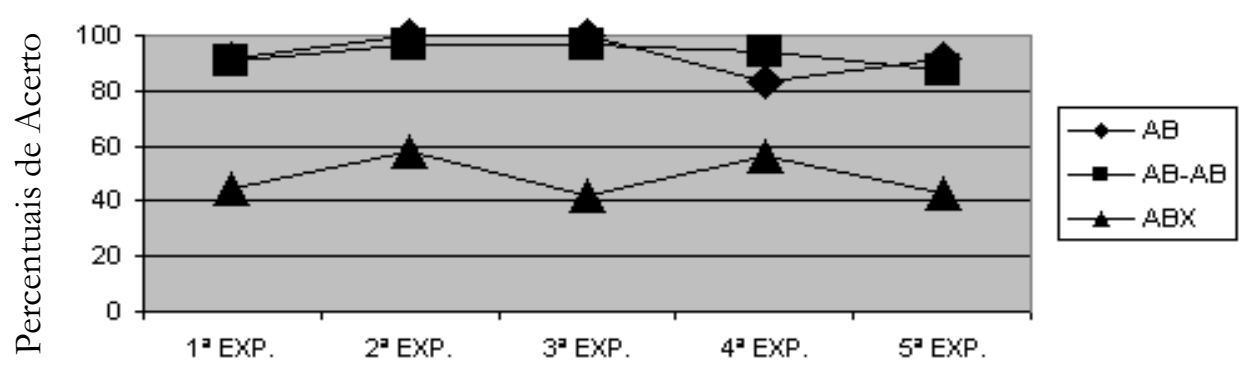

Número de Exposições aos Treinos

Figuras 2. Percentuais de acerto dos Participantes 1, 2 e 3 nos treinos das relações condicionais $\mathrm{AB}, \mathrm{AB}-\mathrm{AB}$ e $\mathrm{ABX}$.

Constata-se que, com a exposição repetida a este procedimento, foram registrados elevados percentuais de acerto no treino das relações condicionais $\mathrm{AB}$, tanto quanto no estabelecimento das relações condicionais de identidade com estímulos complexos AB-AB. Em oposição, os percentuais de acerto mantiveram-se em nível de acaso no treino das quatro relações $\mathrm{ABX}$ durante as exposições repetidas aos respectivos blocos de tentativas. Os resultados insatisfatórios no treino das relações $\mathrm{ABX}$ inviabilizaram a continuidade do experimento, sem a ocorrência, portanto, da exposição dos participantes aos treinos e aos testes previstos na Fase 2.

\section{Discussão}

No presente estudo, três alunos de sala de ensino especial atestaram a aprendizagem de duas relações condicionais (A1B1 e A2B2) com estímulos arbitrários nãorepresentacionais e, em seguida, a emergência imediata das respectivas relações simétricas. Estabeleceram igualmente relações condicionais de identidade envolvendo estímulos (modelo e escolha) complexos (relações A1B1-A1B1, A2B2$\mathrm{A} 2 \mathrm{~B} 2, \mathrm{~A} 1 \mathrm{~B} 2-\mathrm{A} 1 \mathrm{~B} 2$ e A2B1-A2B1), mas registraram resultados negativos no treino de quatro relações condicionais ABX. Neste treino, as funções discriminativas ( $\mathrm{S}+\mathrm{e} \mathrm{S}-$ ) dos 
estímulos de escolha (X1 e X2) deveriam ser definidas pela natureza da relação entre os componentes do estímulo modelo complexo: caso os componentes sustentassem relação de condicionalidade previamente treinada (A1B1 e $\mathrm{A} 2 \mathrm{~B} 2$ ), a seleção de $\mathrm{X} 1$ seria correta; se os componentes não sustentassem tal relação (A1B2 e A2B1), X2 assumia a função de $\mathrm{S}+$.

A princípio, o insucesso na aprendizagem das quatro relações ABX replicou os dados registrados por seis crianças do estudo de Lopes Jr. e colaboradores (2001). Contudo, parece-nos que, em relação a este estudo anterior, informações sobre propriedades do responder condicional com estímulos complexos foram acrescentadas pelo presente trabalho.

Insucessos registrados, em estudos anteriores, na aprendizagem das relações condicionais $\mathrm{ABX}$, admitiam uma descrição em termos do desenvolvimento de relações de controle de estímulo (ou de topografias de controle de estímulo; cf. Mcllvane, Serna, Dube \& R. Stromer, 2000) incompatíveis com aquelas previstas experimentalmente. Em seu conjunto, os dados do presente trabalho viabilizam avaliações adicionais da hipótese de que os resultados negativos na aprendizagem das relações $A B X$ estariam funcionalmente relacionados com a ocorrência do controle restrito de estímulo (seletividade de estímulo, Dube \& Mcllvane, 1997, 1999) manifesto em termos de topografias de controle de estímulo concorrentes.

Segundo Dube e Mcllvane (1999), o procedimento de resposta de observação diferencial pode efetivamente refinar habilidades discriminativas, possibilitando, no caso do presente trabalho, o estabelecimento de um responder discriminado para cada componente do estímulo modelo complexo. Entretanto, R. Stromer e colaboradores (1993a) salientaram que o controle restrito de estímulo foi observado mesmo quando o controle discriminativo foi demonstrado em separado por todos os componentes dos estímulos complexos, precedendo ao treino no qual tais estímulos foram apresentados conjuntamente.

Em Lopes Jr e colaboradores (2001), no Experimento 2 , os resultados negativos no treino das relações $A B X$ poderiam estar funcionalmente relacionados com a ocorrência do controle restrito de estímulo, que manifestouse na exposição ao treino das relações entre os estímulos A e B que definiriam as funções discriminativas ( $\mathrm{S}+$ ou $\mathrm{S}$-) dos estímulos X1 e X2 (treino $\mathrm{ABX}$ ), mas não na aprendizagem das relações FFX e nos testes das relações ZZX. Demonstrou-se assim uma nítida independência funcional: por um lado, a aquisição e a emergência de um responder condicional definido por relações de identidade e de não identidade entre os componentes do estímulo modelo; de outro lado, resultados negativos no ensino de um responder condicional definido por relações de condicionalidade e de não-condicionalidade arbitrárias entre os componentes do estímulo modelo. Em outros termos, o estabelecimento e a emergência do responder condicional definido pela natureza das relações entre os componentes do estímulo modelo complexo variou em função das relações definirem identidade, nãoidentidade, condicionalidade e não-condicionalidade. Considerando-se que a aquisição e a emergência das relações FFX e ZZX, respectivamente, evidenciam ou pressupõem um responder discriminado para cada componente do estímulo modelo e que os resultados negativos do treino das relações $A B X$ expressem medidas comportamentais do controle restrito de estímulo, o Experimento 2 de Lopes Jr. e colaboradores (2001) forneceu evidências adicionais corroborando os argumentos de R. Stromer e colaboradores (1993a). Mas, no Experimento 2 de Lopes Jr. e colaboradores (2001), os estímulos para os quais o responder discriminado foi demonstrado foram aqueles dos conjuntos F (F1 e F2) e Z (Z1 e Z2) e não os estímulos componentes das relações $\mathrm{ABX}$.

Os dados do presente trabalho fortaleceram igualmente os argumentos de R. Stromer e colaboradores (1993), ao demonstrarem resultados negativos no treino das relações $\mathrm{ABX}$ mesmo com evidências que sugerem responder discriminado para cada componente dos estímulos modelos complexos, ou seja, mesmo com os elevados índices de acerto nas respostas de observação diferenciais. Ademais, Dube e Mcllvane (1999) demonstraram que a reincidência do controle restrito de estímulo, para o estabelecimento de relações parciais de identidade, se manifestou por ocasião da retirada do procedimento de resposta de observação diferencial. No presente trabalho, o insucesso diante do treino das relações $A B X$ foi verificado durante a execução do procedimento de resposta de observação diferencial.

Em termos conclusivos, Lopes Jr. e colaboradores (2001) e os dados deste trabalho convergem em apontar a independência funcional dos repertórios discriminativos que definem o treino do responder condicional sobre controle da natureza das relações sustentadas entre os componentes do estímulo modelo. Em termos do delineamento de programas de ensino de habilidades discriminativas, procedimentos efetivos para estabelecer controle condicional fundamentado em relações de identidade e de não-identidade entre os componentes do estímulo modelo podem não garantir, de imediato, extensões de habilidades quando estão envolvidas relações arbitrárias entre estímulos, mesmo diante de evidências que indicam responder discriminado para cada estímulo componente dos estímulos modelos complexos.

Psicologia: Reflexão e Crítica, 2003, 16(1), pp. 71-84 
Assim, um aspecto crítico para as pesquisas futuras que objetivem estabelecer o controle condicional pelas relações entre os componentes do estímulo modelo complexo reside na identificação de variáveis de procedimento funcionalmente relacionadas com as dificuldades registradas na aprendizagem das relações ABX. Para tanto, dados da literatura sobre procedimentos de ensino de habilidades discriminativas certamente cumprirão importante função instrucional (Dube, 1996; Kennedy \& Serna, 1995; Serna \& cols., 1998; Wilkinson \& cols., 1998; Wilkinson \& McIlvane, 1997; Zygmont, Lazar, Dube \& McIlvane, 1992). A investigação e o aperfeiçoamento de metodologias experimentais para o treino de repertórios discriminativos, em particular com estímulos complexos, podem viabilizar significativos avanços, não apenas na compreensão dos processos comportamentais que definem a aprendizagem de relações entre estímulos, mas na proposição de recursos (técnicas, táticas, materiais) que ampliem a eficiência das contingências de ensino de habilidades acadêmicas relevantes.

\section{Referências}

Annet, J. \& Leslie, J. (1995). Stimulus equivalence class involving olfactory stimuli. The Psychological Record, 45, 439-450.

Belanich, J. \& Fields, L. (1999). Tactual equivalence class formation and tactual-to-visual cross modal transfer. The Psychological Record, 49, 75-91.

Burke, J. (1991). Some developmental implications of a disturbance in responding to complex environmental stimuli. American Journal on Mental Retardation, 96, 37-52.

Bush, K., Sidman, M. \& de Rose, T. (1989). Contextual control of emergent equivalence relations. Journal of the Experimental Analysis of Behavior, 51, $29-45$.

Carpentier, F., Smeets, P. \& Barnes-Holmes, D. (2000). Matching compound samples with unitary comparisons: Derived stimulus relations in adults and children. The Psychological Record, 50, 671-685.

Carter, D. \& Werner, T. (1978). Complex learning and information processing by pigeons: A critical analysis. The Journal of the Experimental Analysis of Behavior, 29, 565-601.

Cumming, W. \& Berryman, R. (1965). The complex discriminated operant: Studies of matching to sample and related problems. Em D. I. Mostofsky (Org.), Stimulus generalization (pp. 284-330). Stanford, CA: Stanford University Press.

DeGrandpre, R., Bickel, W. \& Higgins, S. (1992). Emergent equivalence relations between intoreceptive (drug) and exteroceptive (visual) stimuli. Journal of the Experimental Analysis of Behavior, 58, 9-18.

Dube, W. (1996). Teaching discriminations skills to persons with mental retardation. Em C. Goyos, M. A. Almeida \& D. G. Souza (Orgs.), Temas em Educação Especial/Programa de Pós-Graduação em Educação Especiall UFSCAR (pp. 73-96). São Carlos: UFSCAR.

Dube, W., Green, G. \& Serna, R. (1993). Auditory successive conditional discrimination and auditory stimulus equivalence class. Journal of the Experimental Analysis of Behavior, 59, 103-114.

Dube, W. \& McIlvane, W. (1997). Reinforcer frequency and restricted stimulus control. Journal of the Experimental Analysis of Behavior, 68, 303316.

Dube, W. \& McIlvane, W. (1999). Reduction of stimulus overselectivity with nonverbal differential observing responses. Journal of the Experimental Analysis of Behavior, 32, 25-33.
Dube, W., McIlvane, W. \& Green, G. (1992). An analysis of generalized identity matching-to-sample test procedures. The Psychological Record, $42,17-28$.

Dube, W. \& Serna, R. (1998). Re-evaluation of a programmed method to teach generalized identity matching-to-sample. Research in Developmental Disabilities, 19 (4), 347-379.

Green, G. (1990). Differences in developmental of visual and auditoryvisual equivalence relations. American Journal on Mental Retardation, 95, 260-270.

Green, G., Mackay, H., Mcllvane, W., Saunders, R. \& Soraci, S. (1990). Perspectives on relational learning in mental retardation. American Journal on Mental Retardation, 95, 249-259.

Gatch, M. \& Osborne, J. (1989). Transfer of contextual stimulus function via equivalence class development. Journal of the Experimental Analysis of Behavior, 51, 369-378.

Hayes, L., Tilley, K. \& Hayes, S. (1988). Extending equivalence class membership to gustatory stimuli. The Psychological Record, 38, 473-482.

Huguenin, N. \& Touchette, P. (1980). Visual attention in retarded adults: Combining stimuli which control incompatible behavior. Journal of the Experimental Analysis of Behavior, 33, 77-86.

Kennedy, C. \& Laitinen, R. (1988). Second-order conditional control of symmetric and transitive stimulus relation: The influence of order effects. The Psychological Record, 38, 437-446.

Kennedy, C. \& Serna, L. (1995). Emergent relational responding based upon quantity and equivalence. The Psychological Record, 45, 241-260.

Koegel, R. \& Schreibman, L. (1977). Teaching autistic children to respond to simultaneous multiple cues. Journal of Experimental Child Psychology, 24, 299-311.

Litrownik, A., McInnis, E., Wetzel-Pritchard, A. \& Filipelli, D. (1978). Restricted stimulus control and inferred attentional deficits in autistic and retarded children. Journal of Abnormal Psychology, 87, 554-562.

Lopes Jr., J. \& Matos, M. A. (1995). Controle pelo estímulo: Aspectos conceituais e metodológicos acerca do controle contextual. Psicologia: Teoria e Pesquisa, 11(1), 33-39.

Lopes Jr., J., Costa, G., Gonsales, L. \& Golfeto, R. (2001). Aprendizagem e emergência de relações condicionais com estímulos modelos complexos. Em H. Guilhardi, M. B. Madi, P. Queiroz \& M. C. Scoz (Orgs.), Sobre comportamento e cognição: Expondo a variabilidade (Vol. 7) (pp. 401-421). Santo André, SP: Esetec.

Loovas, O., Koegel, R. \& Schreibman, L. (1979). Stimulus overselectivity in autism: A review of research. Psychological Bulletin, 86, 1236-1254.

Loovas, O., Schreibman, L., Koegel, R. \& Rehm, R. (1971). Selective responding by autistic children to multiple sensory input. Journal of Abnormal Psychology, 77, 211-222.

Lynch, D. \& Green, G. (1991). Development and cross modal transfer of contextual control of emergent stimulus relation. Journal of the Experimental Analysis of Behavior, 56, 139-154.

Markham, M. \& Dougher, M. J. (1993). Compound stimuli in emergent stimulus relations: Extending the scope of stimulus equivalence. Journal of the Experimental Analysis of Behavior, 60, 529-542.

McIlvane, W., Dube, W. \& Callahan, T. (1996). Attention: A behavioral analytical perspective. Em G. R. Lyon \& N. Krasnegor (Orgs.), Attention, memory, and executive function (pp. 97-117). Baltimore: Paul H. Brookes.

Mcllvane, W., Serna, R., Dube, W. \& Stromer, R. (2000). Stimulus control topography coherence and stimulus equivalence: Reconciling test outcomes with theory. Em J. Leslie \& D. E. Blackman (Orgs.), Issues in experimental and applied analysis of buman behavior (pp. 85-110). Reno: Context Press.

O'Leary, C. \& Bush, K. (1996). Stimulus equivalence in the tactile modality. The Psychological Record, 46, 589-518.

Pérez-González, L. (1994). Transfer of relational stimulus control in conditional discrimination. Journal of the Experimental Analysis of Behavior 61, 487-503.

Reynolds, G. (1961). Attention in the pigeon. Journal of the Experimental Analysis of Behavior, 4, 203-208. 
Schenk, J. (1993). Emergent conditional discrimination in children: Matching to compound stimuli. The Quarterly Journal of Experimental Psychology, 46B(4), 345-365.

Schreibman, L., Charlop, M. \& Koegel, R. (1982). Teaching autistic children to use extra stimulus prompts. Journal of Experimental Child Psychology, 33, 475-491.

Serna, R., Wilkinson, K. \& McIlvane, W. (1998). Blank-comparison asessment of stimulus-stimulus relations in individuals with mental retardation: A methodological note. American Journal on Mental Retardation, 103(1), 60-74.

Sidman, M. (1986). Functional analysis of emergent verbal classes. Em T. Thompson \& M. Zeiler (Orgs.), Analysis and integration of behavioral units (pp. 213-245). Hillsdale, NJ: Erlbaum.

Sidman, M. (1994). Equivalence relation and behavior: A research story. Boston: Authors Cooperative.

Smeets, P., Schenk, J. \& Barnes, D. (1995). Establishing arbitrary stimulus classes via identity-matching training and non-reinforced matching with complex stimuli. The Quarterly Journal of Experimental Psychology, 48B, 311-328.

Stromer, R., McIlvane, W., Dube, W. \& Mackay, H. (1993a). Assessing control by elements of complex stimuli in delayed matching to sample. Journal of the Experimental Analysis of Behavior, 59, 83-102.

Stromer, R., McIlvane, W. \& Serna, R. (1993b). Complex stimulus control and equivalence. Psychological Record, 43, 585-598.
Stromer, R. \& Stromer, J. (1990a). The formation of arbitrary stimulus classes in matching to complex samples. The Psychological Record, 40, 51 66.

Stromer, R. \& Stromer, J. (1990b). Matching to complex samples: Further study of arbitrary stimulus classes. The Psychological Record, 40, 505-516.

Stromer, R. \& Stromer, J. (1992). Formation of arbitrary stimulus classes in matching to complex samples: Supplementary data. Perceptual and Motor Skills, 75, 505-506.

Tierney, K., DeLangy, P. \& Braken, M. (1995). Formation of an equivalence class incorporating haptic stimuli. The Psychological Record, 45, 431-437.

Wilkinson, K., Dube, W. \& McIlvane, W. (1998). Fast mapping and exclusion (emergent matching) in developmental language, behavior analysis, and animal cognition research. The Psychological Record, 48, 407-422.

Wilkinson, K. \& McIlvane, W. (1997). Blank comparison analysisof emergent symbolic mapping by young children. Journal of Experimental Child Psychology, 67, 115-130.

Zygmont, D., Lazar, R., Dube, W. \& McIlvane, W. (1992). Teaching arbitrary matching via sample stimulus-control shaping to young children and mentally retarded individuals: A methodological note. Journal of the Experimental Analysis of Behavior, 57, 109-117.

Recebido: 23/01/2002

$1^{a}$ revisão: 04/04/2002

Aceite final: $19 / 08 / 2002$

\section{Sobre os autores}

Jair Lopes Junior é Professor Assistente-Doutor do Departamento de Psicologia da Universidade Estadual Paulista (UNESP)/Campus Bauru. É Psicólogo graduado pela Universidade de Brasília, Mestre e Doutor em Psicologia pela Universidade de São Paulo.

Giovana Galvanin da Costa é Graduanda em Psicologia junto à Universidade Estadual Paulista (UNESP)/Campus Bauru e bolsista IC/FAPESP (Proc. 97/12535-1). 